\title{
On the strain-induced fibrillar microstructure of polyethylene: Influence of chemical structure, initial morphology and draw temperature
}

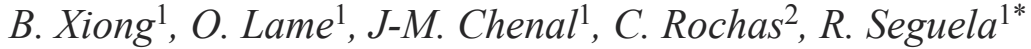 \\ ${ }^{1}$ MATEIS, UMR 5510 CNRS - INSA de Lyon, Batiment Blaise Pascal, Campus LyonTech La Doua, 69621 Villeurbanne, \\ France \\ ${ }^{2}$ CERMAV, UPR 5301 CNRS, BP 53, 38041 Grenoble, France
}

Received 31 July 2015; accepted in revised form 21 October 2015

\begin{abstract}
The influence of crystalline microstructure and molecular topology on the strain-induced fibrillar transformation of semi-crystalline polyethylenes having various chemical structures including co-unit content and molecular weight and crystallized under various thermal treatments was studied by in situ SAXS at different draw temperatures. The long period of the nascent microfibrils, $L_{\mathrm{pf}}$, proved to be strongly dependent on the draw temperature but non-sensitive to the initial crystallization conditions. $L_{\mathrm{pf}}$ was smaller than the initial long period. Both findings have been ascribed to the straininduced melting-recrystallization process as generally claimed in the literature. The microfibrils diameter, $D_{\mathrm{f}}$, was shown to depend on the draw temperature and initial microstructure in a different way as $L_{\mathrm{pf}}$. The evolution of $D_{\mathrm{f}}$ was shown to correlate with the interfacial layer thickness that mainly depends on the chemical structure of the chains. It was concluded that, in contrast to $L_{\mathrm{pf}}$, the microfibril diameter should not be directly sensitive to the strain-induced melting-recrystallization. The proposed scenario is that after the generation of the protofibrils by fragmentation of the crystalline lamellae at yielding, the diameter of the microfibril during the course of their stabilization should be governed by the chain-unfolding and subsequent aggregation of the unfolded chains onto the lateral surface of the microfibrils. The morphogenesis of the microfibrils should therefore essentially depend on the chemical structure of the polymer that governs its crystallization ability, its chain topology and subsequently its fragmentation process at yielding. This scenario is summed up in a sketch.
\end{abstract}

Keywords: thermal properties, polyethylene, microfibrils, crystallinity, crystalline microstructure

\section{Introduction}

A general feature of the morphogenesis of semicrystalline polymers is the regular stacking of chainfolded lamellar crystals alternating with amorphous layers. This stacking $s$ characterized by an intercrystalline long period $L_{\mathrm{p}}$ including the crystalline lamella and amorphous layer thicknesses. The stacking long period is in the range of a few nanometers to a few tens. When crystallization is performed from a quiescent melt, the resulting microstructure is isotropic with a spherulitic arrangement of the lamella stacks as a result of the radial growth of the crystals from a common multiple nucleus often designated as axialite [1]. The size scale of spherulites ranges from a few to several hundred microns.

Upon plastic deformation at large strains, isotropic semicrystalline polymers transform into fibrillar materials that aroused a great interest from both academic and industrial standpoints owing to their remarkable mechanical performances [2-6] to be used as ropes, textile fibers for high performance tissues and fabrics, reinforcing fibers for polymer-based composites, etc.

\footnotetext{
${ }^{\text {*Corresponding author, e-mail: roland.seguela@insa-lyon.fr }}$ (C) BME-PT
} 
The so-called fibrillar microstructure occurs via a plastic instability process, accompanied by a more or less pronounced geometric necking. The fibrillar microstructure consists of bundles of microfibrils parallel to the draw direction, each microfibril being made of a succession of crystal blocs and amorphous layers. It is generally admitted that this microstructure originates from the fragmentation of the crystalline lamellae into blocs during the plastic instability phenomenon [7-11]. These blocks rearrange into strings of pearls thanks to the molecular interconnections generated by the partial unfolding of chains. From small-angle X-ray scattering (SAXS) experiments, it was shown that the crystal blocs are regularly arranged with a long period, $L_{\mathrm{pf}}$, along the microfibril axis that is different from the long period of original isotropic material. For various kinds of semi-crystalline polymers, $L_{\mathrm{pf}}$ proved to be essentially dependent on the draw temperature in a similar way as $L_{\mathrm{p}}$ depends on the temperature of quiescent crystallization from the melt or from solution, i.e. the higher the temperature the greater the long period [7-10]. Considering this complete reconstruction of the stacking long period, many authors claimed that fibrillar transformation necessarily involves a strain-induced melting-recrystallization process [11, 12]. Several phenomena have been proposed for providing physical support to this hypothesis: the chainunfolding mechanism that necessarily goes through a transitory state of disordered chain segments analogous to the amorphous state, the conversion of plastic work into heat that may generate significant temperature increase at high strain rates, the stressinduced depression of the melting point, etc. It was even suggested by Mandelkern and co-workers that the initial morphology was completely erased by the strain-induced melting-recrystallization [13, 14].

In parallel, a few SAXS studies have been performed for characterizing the microfibril transverse dimension or width thanks to a Guinier analysis of the transverse intensity profile of the characteristic 2-streak of the one-dimensional fibrillar stacking [7, 15-20]. In this analysis framework, the transverse intensity profile is considered to be governed by the form factor of the scattering microfibrils. It turned out that both draw temperature and draw ratio, and subsequent annealing treatment as well, have an influence of the microfibril width. However, no correlations were made with structural features of the initial semi-crystalline morphology.
The above remarks regarding the fibrillar morphology contrast with the numerous studies dealing with the incidence on the elementary mechanisms of plastic deformation of the initial morphology and molecular topological of semi-crystalline polymers, such as crystallinity, crystal thickness, chain entanglement and tie chain densities, etc. together with deformation conditions [21-36]. It was shown in some cases that these structural or experimental factors could have an impact on the mechanism of fragmentation of the crystalline lamella via the local stress distribution on the crystalline lamellae $[22,25$, $30]$. So that one may foresee some consequences on the microfibril morphology in spite of the meltingrecrystallization process.

In a previous paper [37] we reported an in situ SAXS study of the fibrillar transformation at room temperature of a series of polyethylene materials covering a wide range of crystallinity $0.48<X_{\mathrm{c}}<0.80$ and long period $17 \mathrm{~nm}<L_{\mathrm{p}}<37 \mathrm{~nm}$. It was shown that though the fibrillar long period did not depend on the initial morphology, the width of the microfibrils displayed a correlation with the initial semi-crystalline morphology. It was suggested that the microfibril width was related to the average size of the initial crystal fragments via the formation of protofibrils that retaind the memory of the crystallization conditions via the density of tie chains and chain entanglements that might survive the strain-induced melting-recrystallization.

The present study is an extension over a large temperature range of our former work of the post-yield drawing of PE. In contrast to most of the previous works dealing with ex situ analysis of fibrillar structure at room temperature, SAXS measurements were carried out in situ during drawing in order to prevent eventual post-drawing crystallization and topological rearrangements. This is likely to provide information closely related to the morphogenesis of the microfibrils during drawing and help understanding the mechanical behavior of the drawn materials in relation to their processing and crystallization conditions. The morphological changes that occur during ultra-drawing beyond the fibrillation stage [4] are not discussed in this work.

\section{Experiment}

\subsection{Materials and sample preparation}

Four linear polyethylenes with different hexene counit contents and very close molecular weights 
were provided by Total Petrochemicals (Feluy, Belgium). The molecular characteristics are given in Table 1. It is worth noticing that is spite of their very close $M_{\mathrm{n}}$ and $M_{\mathrm{w}}$ values and similar co-units contents, PE-C and PE-D have significantly different $M_{z}$ values. This is relevant to the presence of a small amount of very long chains in PE-D that is expected to influence the crystallization behavior $[38,39]$.

The four polymers have been submitted to three different thermal treatments in order to modify their crystalline microstructure and chain-topology. Quenching is expected to keep the entanglement topology of the melt and generate thin crystals. In contrast, annealing in the solid state and isothermal crystallization from the melt are expected to reduce the entanglement density and generate thick crystals, the latter treatment being more efficient than the former one as evidenced through the data of Table 2 . The polymer pellets were compression-molded at $170^{\circ} \mathrm{C}$ into $500 \mu \mathrm{m}$ sheets between aluminum foils in a heating press and then quenched into water at room temperature (RT). These samples were called 'quenched'. Some of the quenched samples were heat treated via two different ways in order to modify the crystalline microstructure. The samples labeled 'annealed' were heated up to the temperature $T_{\mathrm{c}}=122^{\circ} \mathrm{C}$ for 15 hours and subsequently slowly cooled down to RT (see $T_{\mathrm{c}}$ determination below). The samples called 'isotherm' were melted at $170^{\circ} \mathrm{C}$ and then cooled in a thermostatic oil bath at the temperature $T_{\mathrm{c}}$ and held at this temperature for 15 hours. More details on the sample preparation are given elsewhere [37, 40].

\subsection{Differential scanning calorimetry (DSC)}

Thermal analysis was conducted on a DSC7 apparatus from Perkin-Elmer using samples of 5-7 mg. The temperature and heat flow scales were calibrated using high purity indium. The heating and cooling scans were recorded at a rate of $10^{\circ} \mathrm{C} / \mathrm{min}$. The crystal weight fraction, $X_{\mathrm{c}}$, was computed from the melting enthalpy of the samples, using the value $\Delta H_{\mathrm{m}}{ }^{\circ}=290 \mathrm{~J} / \mathrm{g}$ for the heat of fusion of perfectly

Table 1. Molecular characteristics of the row PE materials

\begin{tabular}{|c|c|c|c|c|}
\hline Material & $\begin{array}{c}\text { Co-unit } \\
{[\mathbf{m o l} \% \mathbf{l}]}\end{array}$ & $\begin{array}{c}\boldsymbol{M}_{\mathbf{n}} \\
{[\mathbf{k g} / \mathbf{m o l}]}\end{array}$ & $\begin{array}{c}\boldsymbol{M}_{\mathbf{w}} \\
{[\mathbf{k g} / \mathbf{m o l}]}\end{array}$ & $\begin{array}{c}\boldsymbol{M}_{\mathbf{Z}} \\
{[\mathbf{k g} / \mathbf{m o l}]}\end{array}$ \\
\hline PE-A & 1.8 & 14.3 & 231 & 2770 \\
\hline PE-B & 0.8 & 15.8 & 187 & 1770 \\
\hline PE-C & 0.1 & 15.4 & 216 & 2770 \\
\hline PE-D & 0.1 & 15.0 & 229 & 4100 \\
\hline
\end{tabular}

crystalline PE [41]. The standard deviation of $X_{\mathrm{c}}$ data is about $1 \%$.

\subsection{SAXS measurements}

Small-angle X-ray scattering (SAXS) experiments were performed on the BM02 beamline of the European Synchrotron Radiation Facility (Grenoble, France) using a wavelength $\lambda=0.154 \mathrm{~nm}$. The 2DSAXS patterns were recorded on a CCD camera from Princeton Instruments (Trenton NJ, USA) which was set at sample-detector distance of $158 \mathrm{~cm}$. In situ recording of SAXS patterns during uniaxial tensile testing was performed using a home-made tensile stage allowing symmetric stretching of the sample. This ensured that all data were recorded from the same location on the sample during deformation. The dumbbell-shaped samples were $11 \mathrm{~mm}$ in gauge length, $4 \mathrm{~mm}$ in width and $0.5 \mathrm{~mm}$ in thickness. The nominal or engineering strain is defined as $\varepsilon=$ $\left(L-L_{0}\right) / L_{0}$ with $L_{0}$ and $L$ the sample gauge length before and during tensile drawing. The strain rate was $6.4 \cdot 10^{-4} \mathrm{~s}^{-1}$ to make sure that the strain increment was less than $0.1 \%$ during the $5 \mathrm{~s}$ time for every pattern recording. The experiments were performed at several draw temperatures in the range $25^{\circ} \mathrm{C}<T_{\mathrm{d}}$ $<100^{\circ} \mathrm{C}$ for each sample.

The Lorentz-corrected intensity profiles of the isotropic samples prior to straining were obtained by azimuthal integration of the $2 \mathrm{D}$ patterns according to Equation (1):

$I(q)=q_{2} \int_{0}^{2 \pi} I(q, \varphi) \mathrm{d} \varphi$

where $\varphi$ is the azimuthal angle. The average long period of the lamellar stacking, $L_{\mathrm{p}}$, was then determined from the Bragg's relation (Equation (2)):

$L_{\mathrm{p}}=\frac{2 \pi}{q_{\max }}$

where $q_{\max }$ is the scattering vector at the maximum of the correlation peak as determined by a Gaussian fitting. The standard deviation of the $L_{\mathrm{p}}$ data does not exceed $1.0 \mathrm{~nm}$.

The crystal thickness, $L_{\mathrm{c}}$, of the isotropic samples was computed from Equation (3):

$L_{\mathrm{c}}=L_{\mathrm{p}} X_{\mathrm{c}} \frac{\rho}{\rho_{\mathrm{c}}}$ 
where $\rho_{\mathrm{c}}=1.00 \mathrm{~g} / \mathrm{cm}^{3}$ is the density of the crystalline phase. This relation assumes much larger length and width of the crystalline lamellae as compared with thickness.

Regarding the drawn samples displaying the characteristic two-streak meridian pattern, the onedimension intensity profile along the meridian was obtained by projection on the meridian of the 2Dpattern according to Stribeck's method [19]. Then the long period along the draw axis, $L_{\mathrm{pf}}$, was estimated by using Equation (2).

The structural data of all isotropic PE samples at RT are reported in Table 2. Also are reported the weight fraction of interfacial zone between crystal and amorphous regions as assessed from Raman spectroscopy, $X_{\mathrm{i}}$, the concentration of stress transmitters $[S T]$ according to Brown-Huang's theoretical approach, and the natural draw ratio at $\mathrm{RT}, \lambda_{\mathrm{N}}$, as already determined in a previous study via in situ mechanical testing $[38,42]$.

A convenient way for quantifying the microfibril width is to analyze the transverse intensity profile of the lobes of the 2-streak SAXS patterns. The scattering objects were supposed to be individually embedded in a medium of lower electron density consisting of amorphous chains and cavities, without interference between them. The corresponding model consists of either elongated cylinders or discs. In both instance, the analysis of the transverse intensity profile of the streak-like lobes can be performed via Guinier's approximation of the form factor leading to an estimation of the transverse dimension of the scattering elements. In the case of scattering cylinders, the relevant Guinier's approximation function is given by Equation (4) [43]:
$I(q)=I_{0} \exp \left(-\frac{q^{2} D_{\mathrm{f}}^{2}}{4}\right)$

where $D_{\mathrm{f}}$ is the cylinder diameter with $D_{\mathrm{f}}=2 R_{\mathrm{f}}$ where $R_{\mathrm{f}}$ is the radius of gyration. Fitting the experimental intensity data with Equation (4) enabled computing only one diameter value for each sample as demonstrated in the previous study [37]. The standard deviation of the $D_{\mathrm{f}}$ data was about $1.0 \mathrm{~nm}$ or less.

\section{Results}

All the SAXS data regarding the fibrillar structure of the materials in the present work are relevant to samples drawn at the same engineering strain $\varepsilon=1.3$. At this strain level the fibrillar transformation has not reached the whole sample length but the necking process is stabilized at the so-called natural draw ratio, $\lambda_{\mathrm{N}}$, and propagates along the sample $[44,45]$. This enables to study the nascent fibrillar structure of each material, irrespective of its macroscopic behavior.

Firstly, it is to be noticed that the $\lambda_{\mathrm{N}}$ data of Table 2 vary over a very large range and do not display correlation with either $X_{\mathrm{c}}$ or $L_{\mathrm{p}}$. On the other hand, the $\lambda_{\mathrm{N}}$ data depend on the thermal treatment for every PE type. This is an evidence that the microfibrillar structure does depend on the initial crystalline microstructure is not thoroughly erased by the strain-induced melting recrystallization process. This strong correlation enabled using $\lambda_{\mathrm{N}}$ as an indicator of the longterm mechanical properties via the correlation with the initial molecular topology $[46,47]$.

Figure 1 shows the typical shape of a SAXS pattern recorded from the quenched PE-A after necking at

Table 2. Structural and physical characteristics of the isotropic PE samples at RT

\begin{tabular}{|c|c|c|c|c|c|c|c|}
\hline Materials & & $\begin{array}{c}X_{\mathrm{c}} \\
{[\%]}\end{array}$ & {$[S T]$} & $\begin{array}{c}X_{\mathbf{i}} \\
{[\%]}\end{array}$ & $\lambda_{\mathrm{N}}$ & $\begin{array}{c}L_{\mathrm{p}} \\
{[\mathrm{nm}]}\end{array}$ & $\begin{array}{c}L_{\mathrm{c}} \\
{[\mathrm{nm}]}\end{array}$ \\
\hline \multirow{3}{*}{ PE-A } & quenched & 48 & 0.24 & 13 & 3.5 & 17 & 8 \\
\hline & annealed & 52 & 0.17 & 14 & 4.0 & 23 & 11 \\
\hline & isotherm & 53 & 0.15 & 11 & 4.7 & 24 & 12 \\
\hline \multirow{3}{*}{ PE-B } & quenched & 54 & 0.19 & 8 & 4.2 & 19 & 9 \\
\hline & annealed & 62 & 0.14 & 8 & 4.7 & 22 & 13 \\
\hline & isotherm & 65 & 0.09 & 4 & 5.5 & 26 & 16 \\
\hline \multirow{3}{*}{ PE-C } & quenched & 65 & 0.19 & 5 & 4.5 & 20 & 12 \\
\hline & annealed & 73 & 0.08 & 5 & 6.0 & 30 & 21 \\
\hline & isotherm & 75 & 0.03 & 5 & 7.5 & 36 & 26 \\
\hline \multirow{3}{*}{ PE-D } & quenched & 69 & 0.17 & 3 & 5.0 & 22 & 14 \\
\hline & annealed & 77 & 0.08 & 5 & 5.7 & 30 & 22 \\
\hline & isotherm & 80 & 0.03 & - & 7.0 & 37 & 29 \\
\hline
\end{tabular}


$T_{\mathrm{d}}=50^{\circ} \mathrm{C}$. This figure schematically shows the methodology of calculation of the microfibril long period $L_{\mathrm{pf}}$ and diameter $D_{\mathrm{f}}$. The strong central scattering extending in equatorial direction arises from interfibrillar cavities or low density regions [19]. The electron density contrast between these regions and the microfibrils is so high that this scattering can turn extremely intense and merge onto the streak-like scattering, particularly for high crystallinity materials. In this instance, the analysis of the structural characteristics of the microfibrils is prohibited. However increasing the draw temperature reduces the propensity for cavitation or interfibrillar density fluctuations [48] so that more samples can be inves- tigated during drawing at elevated temperature than in the case of the drawing at RT. This is one of the major benefits from the present study compared to our previous one [37].

Figure 2 shows the evolution of the meridian intensity profile of quenched PE-A as a function of strain at two different $T_{\mathrm{d}}$. The SAXS patterns change from isotropic to highly anisotropic patterns with two streak-like scattering patterns during the elongation of samples.

The central SAXS patterns of Figures $2 a$ and $2 b$ show the SAXS patterns about the onset of the streaklike reflections characteristic of the fibrillar structure, for both $T_{\mathrm{d}}$. The actual strain onset decreases
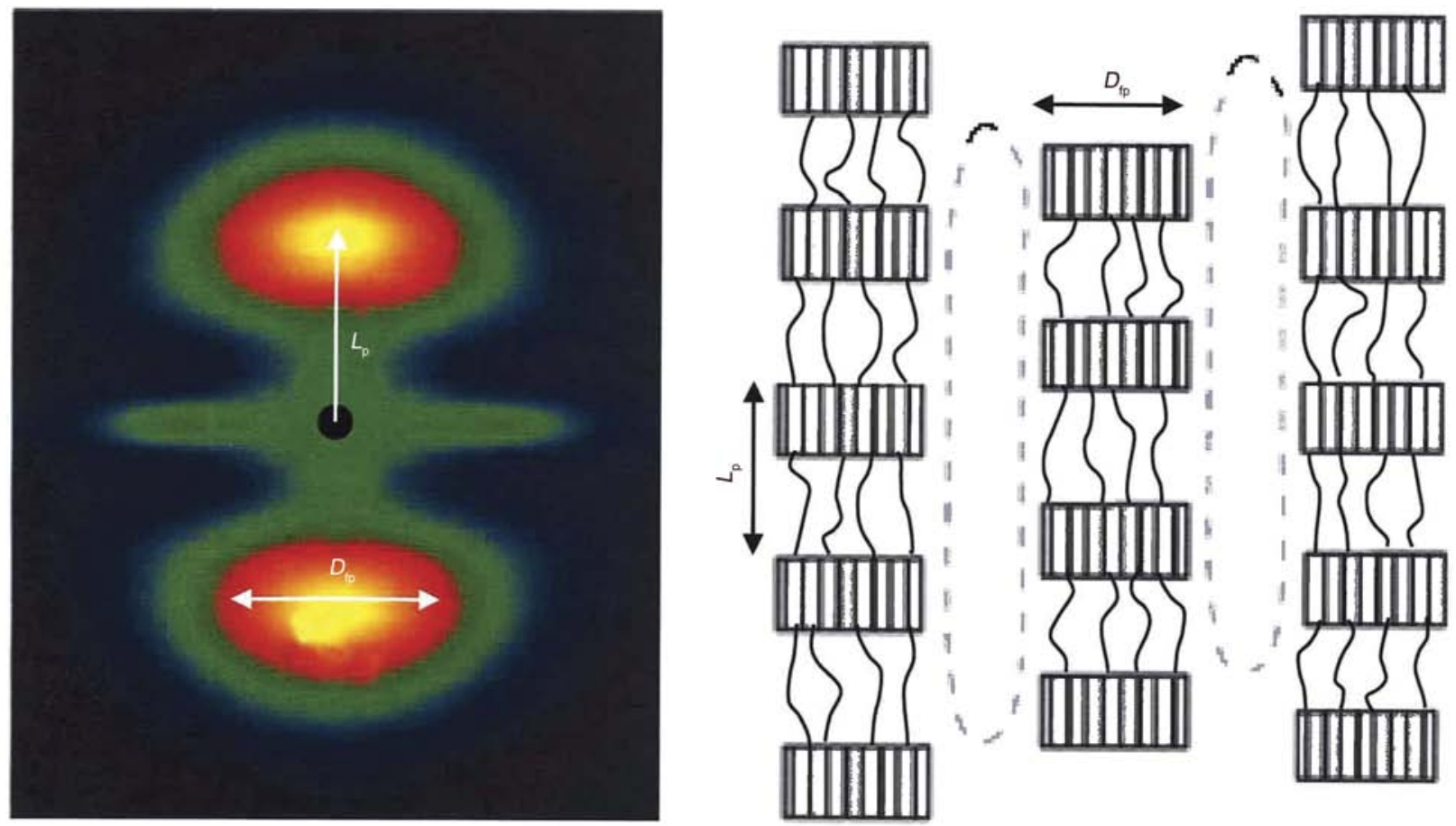

Figure 1. Schematic SAXS pattern and molecular model of the fibrillar microstructure (borrowed from Stribeck ref. 19)
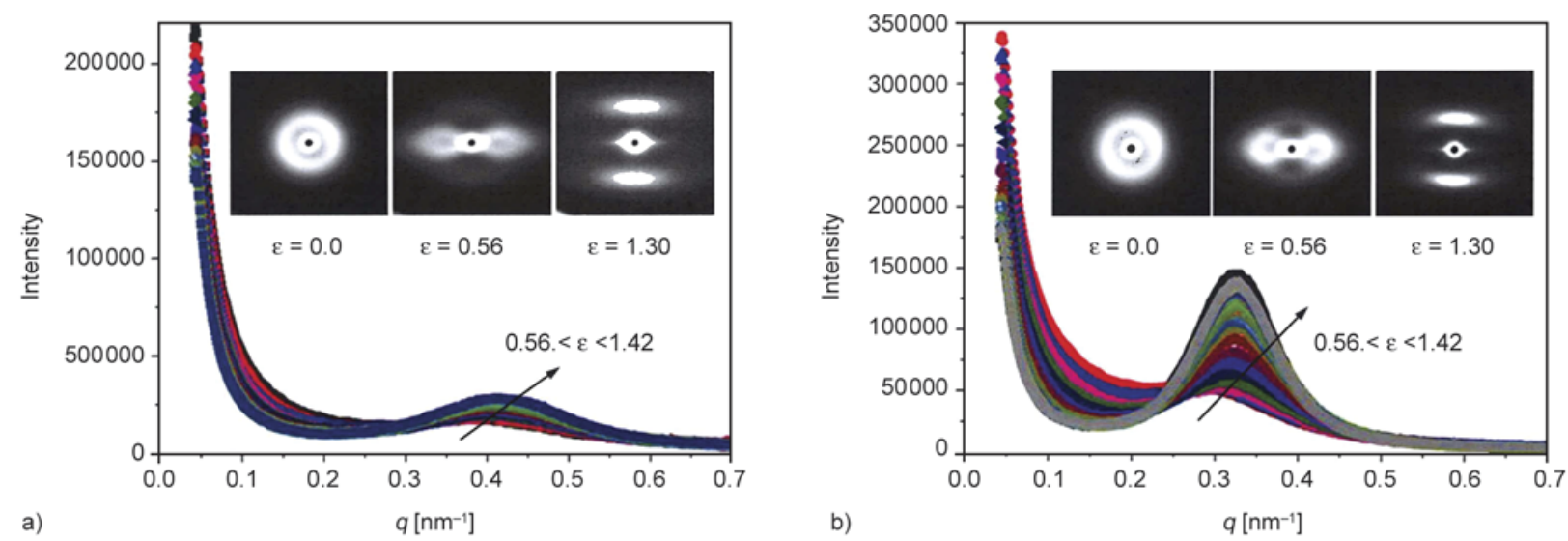

Figure 2. SAXS patterns and meridian intensity profile of quenched PE-A as a function of strain : (a) $T_{\mathrm{d}}=50^{\circ} \mathrm{C}$; (b) $T_{\mathrm{d}}=100^{\circ} \mathrm{C}$ 
with increasing $T_{\mathrm{d}}$ suggesting that the fully orientated structure forms faster at higher $T_{\mathrm{d}}$. The generation of microfibrils from the crystalline lamella fragmentation should indeed be strongly promoted at higher $T_{\mathrm{d}}$ due to the temperature dependence of the crystal stiffness in the temperature domain of the crystal mechanical relaxation. Besides, at a given $T_{\mathrm{d}}$, the streak-like scattering intensity strongly increases during elongation due to the gradual conversion of lamellae into microfibrils, and the gradual improvement of orientation as well.

Figure 3 shows $L_{\mathrm{pf}}$ as a function of initial $L_{\mathrm{p}}$ for all samples at different $T_{\mathrm{d}}$. At low $T_{\mathrm{d}}$, annealed and isotherm samples could not be quantitatively analyzed due to the merging of the intense void scattering onto the microfibrillar scattering. However, more $L_{\mathrm{pf}}$ data could be obtained when elevating $T_{\mathrm{d}}$ due to the reduced propensity of the samples for cavitation, as judged from the significant decrease of the scattering about the beamstop (Figure 2).

Figure 3a shows that $L_{\mathrm{pf}}$ is generally smaller than the initial $L_{\mathrm{p}}$ indicating dramatic reconstruction of the periodic stacking of amorphous and crystalline phases as a result of the plastic deformation. If considering the SAXS intensity profiles of Figure 2, this phenomenon seems to be rather gradual as a function of increasing strain. In addition, $L_{\mathrm{pf}}$ increases with increasing $T_{\mathrm{d}}$. These findings are in perfect agreement with the melting-recrystallization concept already observed for various semi-crystalline polymers [49-51].

The roughly linear increase of $L_{\mathrm{pf}}$ as a function of initial $L_{\mathrm{p}}$, for every $T_{\mathrm{d}}$ value, may be assigned to the

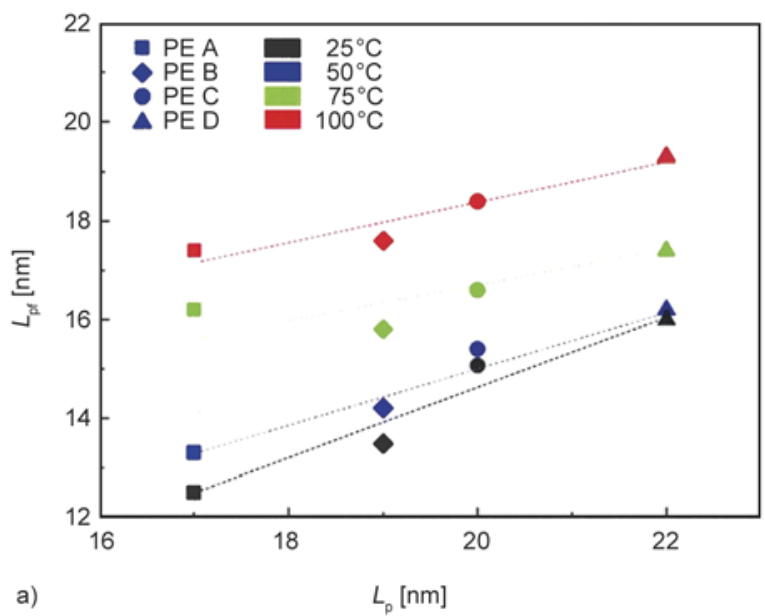

crystallization potential of the various PEs, i.e. a chemical structure dependence including counit content and molecular weight. The chemical structure of the chains indeed governs in a similar way the generation of $L_{\mathrm{p}}$ from the quiescent crystallization in the melt and that of $L_{\mathrm{pf}}$ from the strain-induced recrystallization upon drawing.

It appears from Figure $3 \mathrm{a}$ that the $L_{\mathrm{pf}}$ data at $\mathrm{RT}$ (black symbols) are surprisingly close to and even overlap the $L_{\mathrm{pf}}$ data at $50^{\circ} \mathrm{C}$ (blue symbols). This apparent insensitivity of the fibrillar long period to the draw temperature in the range $25^{\circ} \mathrm{C}<T_{\mathrm{d}}<50^{\circ} \mathrm{C}$ has been formerly reported by Peterlin and BaltaCalleja for PE materials covering a similar $X_{\mathrm{c}}$ range as the present one and for a similar $T_{\mathrm{d}}$ domain [22]. These authors conjectured that the roughly invariant behavior of $L_{\mathrm{pf}}$ for $T_{\mathrm{d}}<50^{\circ} \mathrm{C}$ can be assigned to the super-cooling effect consecutive to the straininduced self-heating and melting of the fibrillated material in the destruction zone during the course of the fibrillar transformation. The reason would be that the crystallization rate turns so high with decreasing temperature that recrystallization of the molten material is almost complete before the actual temperature of the sample reaches the experimental $T_{\mathrm{d}}$ value. It results that $L_{\mathrm{pf}}$ gradually turns $T_{\mathrm{d}}$-independent with decreasing $T_{\mathrm{d}}$.

Regarding Figure 3b, samples of the same PE material with different crystalline microstructures generated by thermal treatments exhibit very small difference in $L_{\mathrm{pf}}$ at given $T_{\mathrm{d}}$, in the range of experimental accuracy. This finding is an additional evidence of the strain-induced melting-recrystallization process

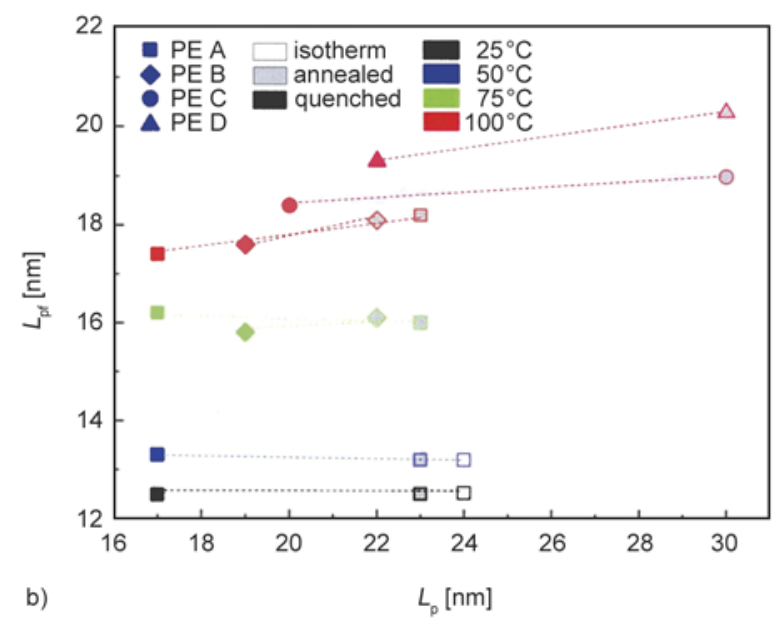

Figure 3. Microfibril long period versus initial $L_{\mathrm{p}}$ at various $T_{\mathrm{d}}$ : (a) influence of chemical structure for quenched samples; (b) influence of thermal treatment (full colors refer to draw temperatures; symbol shapes refer to the various polymers, declining tint of every color refers to thermal treatments) 

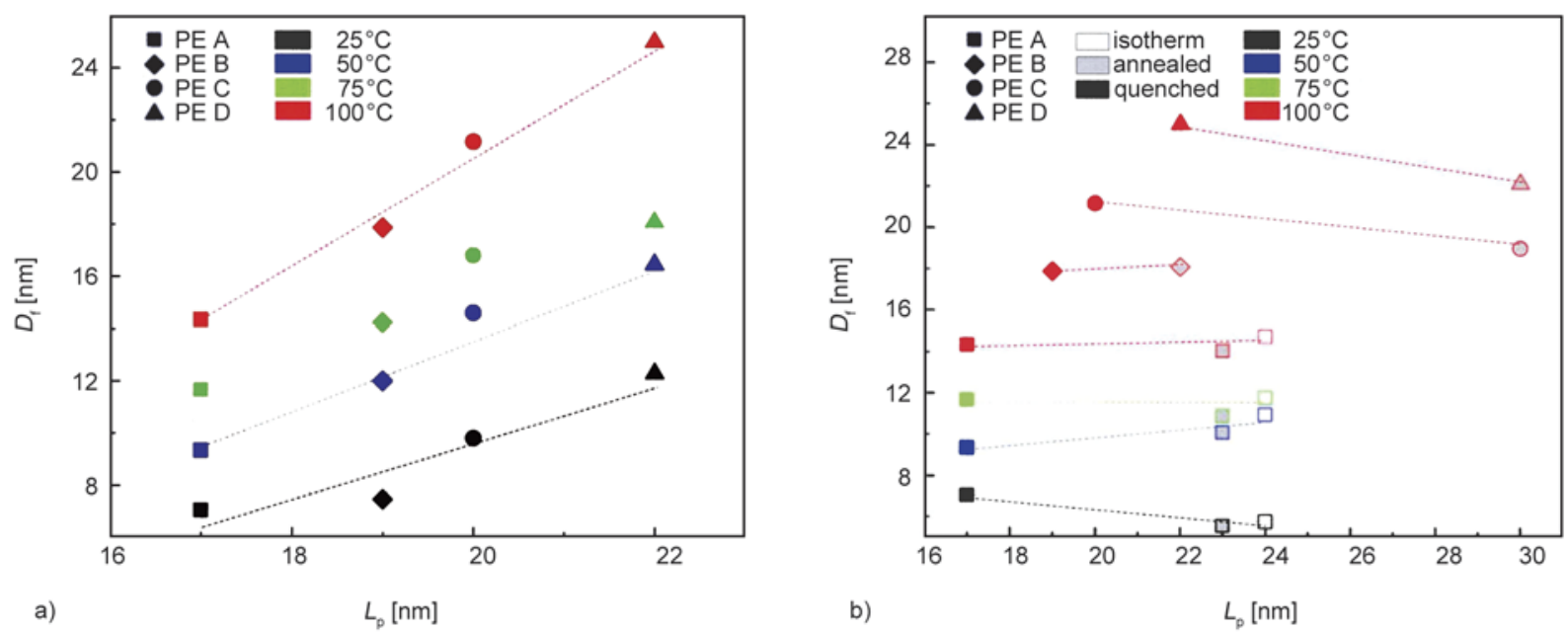

Figure 4. Microfibril diameter versus stacking long period: (a) influence of chemical structure for the quenched samples; (b) influence of thermal treatment

that completely erases the initial crystalline microstructure, from the standpoint of crystal stacking in the microfibrils.

Figure 4 shows the microfibril diameter as a function of long period at different $T_{\mathrm{d}}$. Similar trends could be obtained if using $D_{\mathrm{f}}$ versus $L_{\mathrm{p}}$. The $D_{\mathrm{f}}$ values perfectly agree with transmission electron microscopy measurements by Tarin and Thomas for high density PE crystallized from the melt under similar conditions [45]. Furthermore, the $D_{\mathrm{f}}$ data of Figure $4 \mathrm{a}$ exhibit a linear variation with $L_{\mathrm{p}}$ at a given $T_{\mathrm{d}}$, that is an evidence of a chemical structure dependence of $D_{\mathrm{f}}$ similar to that of $L_{\mathrm{pf}}$.

Regarding the $D_{\mathrm{f}}$ evolution with thermal treatment in Figure $4 \mathrm{~b}$ for a given PE type and for a given $T_{\mathrm{d}}$, it appears a faint increase with increasing $L_{\mathrm{p}}$, i.e. from quenched to annealed and isotherm sample. The above observations suggest that $D_{\mathrm{f}}$ is slightly correlated to the initial crystalline microstructure. But the most striking feature of Figure 4 is that $D_{\mathrm{f}}$ is temperature-dependent over the whole $T_{\mathrm{d}}$ range, in contrast to $L_{\mathrm{pf}}$ that no longer depends on temperature for $T_{\mathrm{d}} \leq 50^{\circ} \mathrm{C}$. This latter finding lets us foreseeing that $D_{\mathrm{f}}$ is influenced by the melting-recrystallization process in the same way as $L_{\mathrm{pf}}$ is. Notwithstanding no global correlation appears between $D_{\mathrm{f}}$ and $L_{\mathrm{p}}$ which is a parameter characteristic of the initial microstructure.

So an attempt has been made to correlate $D_{\mathrm{f}}$ to a parameter characteristic of the chain topology. Figure 5 shows $D_{\mathrm{f}}$ as a function of the natural draw ratio $\lambda_{\mathrm{N}}$ which is a macroscopic indicator of the concentration of stress transmitters $[S T]$ as deduced from Brown-Huang's model [40, 42]. The reason for

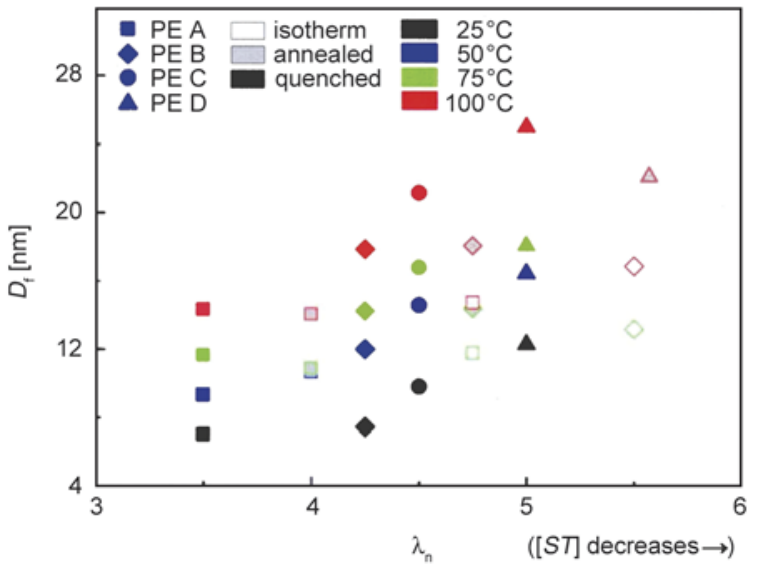

Figure 5. Microfibril diameter versus natural draw ratio at different temperatures

choosing a macroscopic indicator $\lambda_{\mathrm{N}}$ instead of the actual molecular parameter $[S T]$ is that the occurrence of large scale chain rearrangements such as chain reeling together with regular chain-folding during crystallization at high temperature of highly crystalline PEs likely decreases the occurrence of tie chains and chain entanglements. This phenomenon is not taken into account in the [ST] computation that is performed in the framework of Fischer's model assuming that the global chain coil conformation of the molten state is preserved in the solid state. This point will be further argued in the Concluding Discussion. In contrast, $\lambda_{\mathrm{N}}$ is an experimental parameter that does account for the topological modifications occurring during crystallization. So only the variation direction of $[S T]$ is shown on the $x$-axis of Figure 5 for the sake of the discussion.

Figure 5 shows that, at a given temperature, the $D_{\mathrm{f}}$ data for the samples having the same thermal treat- 
ment linearly increase with increasing $\lambda_{\mathrm{N}}$ and decreasing [ST]. This is again in agreement with the chemical structure dependence. Quenched samples exhibit smaller $D_{\mathrm{f}}$ than annealed and isothermal samples. However, $D_{\mathrm{f}}$ shows little if any dependence on thermal treatment for a given PE material. There is again no global correlation between $D_{\mathrm{f}}$ and the topological parameter, $\lambda_{\mathrm{N}}$ or $[S T]$, as it was the case for the structural parameter $L_{\mathrm{p}}$.

Alternatively, we have tried a mixed correlation with crystalline microstructure and chain topology by using Nitta's parameter, $L_{\mathrm{c}} /[S T]^{0.5}$ [52]. Nitta's approach considers yielding as a fragmentation process, the size of the crystal fragment being basically determined by the average distance between tie chains. These topological features are the locus of stress concentration that initiates the lamella fragmentation. From a mechanical standpoint, the $L_{\mathrm{c}}$ factor in this parameter can be seen as featuring the shear stress to which it is roughly proportional according to the dislocation theory at the onset of the lamellae fragmentation [23]. The $1 /[S T]^{0.5}$ factor accounts for the distribution of stress on the lamella surface via the density of the stress transmitters. The unreported data displayed no clear correlation between $D_{\mathrm{f}}$ and $L_{\mathrm{c}} /[S T]^{0.5}$ for the various $T_{\mathrm{d}}$ values of the present experiments. This is in agreement with our previous findings at RT.

Finally a correlation was attempted with the interfacial layer thickness which worked fairly well at RT [37]. Figure 6 shows the $D_{\mathrm{f}}$ versus $X_{\mathrm{i}}$ plots at various $T_{\mathrm{d}}$. For a given $T_{\mathrm{d}}$ value, all the $D_{\mathrm{f}}$ data fall on the same curve, irrespective of the thermal treatment. Moreover, this finding applies to every temperature, each curve being shifted upward with increasing $T_{\mathrm{d}}$.

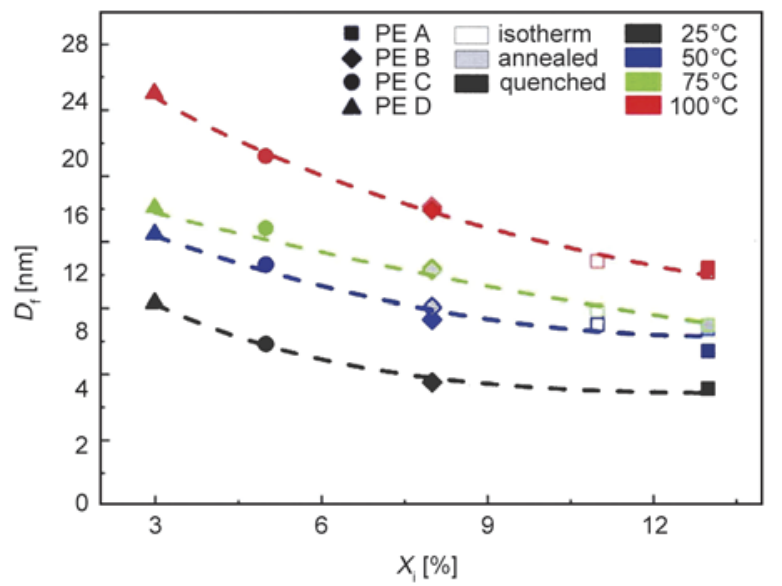

Figure 6. Microfibril diameter versus interfacial layer thickness at different draw temperatures
Worth noticing is that $X_{\mathrm{i}}$ is not much dependent on the thermal treatment, but rather on the chemical structure of the polymer. So that it is not clear whether $D_{\mathrm{f}}$ actually depends on the initial crystalline microstructure and chain topology of the samples via $X_{\mathrm{i}}$ or depends on the chemical structure of the polymer in a similar way as $X_{\mathrm{i}}$ (see Table 1 ).

\section{Conclusions}

Two major findings have been disclosed regarding the microfibril structure. First, $D_{\mathrm{f}}$ depends on $T_{\mathrm{d}}$ over the whole temperature range $25^{\circ} \mathrm{C}<T_{\mathrm{d}}<100^{\circ} \mathrm{C}$, in strong contrast with $L_{\mathrm{pf}}$ that levels off for $T_{\mathrm{d}} \leq 50^{\circ} \mathrm{C}$. This can be taken as an evidence that the microfibril diameter and long period do not depend in a similar way on the strain-induced meltingrecrystallization process. Second, $D_{\mathrm{f}}$ obeys a unique correlation with $X_{\mathrm{i}}$ at every $T_{\mathrm{d}}$ value. This suggests that $D_{\mathrm{f}}$ mainly depends on the mechanism of lamella fragmentation at the onset of yielding, the correlation with $X_{\mathrm{i}}$ being the footprint of the chain topology on the lamella fragmentation process.

However, it is quite surprising that $D_{\mathrm{f}}$ steadily increases with decreasing $X_{\mathrm{i}}$ in parallel to an increase of $\lambda_{\mathrm{N}}$ as observed in Figure 5. Indeed a greater $\lambda_{\mathrm{N}}$ value is indicative of a greater drawability of the microfibrils, namely a high potentiality for chainunfolding. Two mechanisms may be proposed in consideration that high crystallinity PEs develop regular chain-folding during crystallization from the quiescent melt due to the reeling phenomenon. In the case of samples having low $X_{\mathrm{i}}$ that goes along with high crystallinity (Table 2 ), lamellae may initially break into smaller crystal fragments owing to the high rate of regular chain-folding so that the microfibrils could develop to greater length according to Peterlin's string of pearls model. Thereby, their diameter should gradually be reduced at constant crystal volume fraction. Alternatively, the crystal fragments in the protofibrils may display greater capability for chain-unfolding resulting in reduced $D_{\mathrm{f}}$ in the final microfibrils. Both these issues should in principle result in a reduction of $D_{\mathrm{f}}$ which contrasts with the observed increase of $D_{\mathrm{f}}$.

For reconciling the concomitant increases of $D_{\mathrm{f}}$ and $\lambda_{\mathrm{N}}$, one may assume that the chains unfolded during the generation of the microfibrils are able to aggregate onto the lateral surface of the protofibrils. By this process, the final $D_{\mathrm{f}}$ of the microfibrils may grow significantly bigger that the initial $D_{\mathrm{f}}$ of the proto- 
fibrils extracted for the fragmented lamellae. Moreover, this process should be more effective for highly crystalline PEs having small co-unit content and high ability for chain unfolding.

This explanation also applies to the observation that $D_{\mathrm{f}}$ increases with increasing $T_{\mathrm{d}}$ at a given $X_{\mathrm{i}}$ value (Figure 6): indeed, the increase of chain mobility with increasing temperature in the present $T_{\mathrm{d}}$ range should promote the gathering of unfolded chains onto the lateral surface of the microfibrils resulting in bigger $D_{\mathrm{f}}$.

To sum up, the morphogenesis of the microfibrils should be essentially dependent on the chemical structure of the polymer that governs its initial crystallization ability and its chain topology and the various stages of the microfibril construction.

At the light of the present data, Peterlin's scenario for the fibrillar transformation may be modified regarding the morphogenesis of the microfibrils as shown in Figure 7, disregarding the classical plastic processes in the crystal such as crystal shear, phase transition and twinning:

- stage 1: protofibrils are generated like string of pearls via fragmentation of lamella stacks and subsequent extraction of the crystal fragments via chain-unfolding from the fracture surfaces of the crystal lamellae. The remaining chain-folded crystal fragments undergo a gradual strain-induced melting owing to the self-heating generated by the plastic work or energy delivered by the partial destruction of the crystals via chain-unfolding. The presence of these remaining chain-folded crystal fragments is due to the more or less irregular chain-topology at the crystal/amorphous interface $\left(X_{\mathrm{i}}\right)$ that prevents or at least hinders chainunfolding. These topologically coherent entities are the ones that preserve the protofibril integrity in spite of strain-induced melting. The protofibril should not be as perfectly oriented as shown on the picture and their uniaxial orientation must develop gradually during the protofibril buildup.

- stage 2: benefiting from the self-heating, the partly molten protofibrils undergo elongation and thinning down thanks to further chain-unfolding up to the natural draw ratio. Then, the chain-unfolding potential of the topologically coherent entities is exhausted. The concomitant strain-hardening entails the stopping of plastic deformation. Selfheating stops and the local drop of temperature enables recrystallization of the protofibrils into incipient microfibrils with new long period. At this stage, incipient microfibrils and protofibrils may coexist for a while.

- stage 3: the completion of recrystallization within the microfibrils together with aggregation and additional crystallization of unfolded chains along the microfibrils finally leads to wider and stable microfibrils. The diameter of the stable microfibrils strongly depends on the material and $T_{\mathrm{d}}$ : the higher are the crystallization potential and $T_{\mathrm{d}}$, the higher is $D_{\mathrm{f}}$. An interfibrillar amorphous phase consisting of non-crystallizable unfolded chains should remain due to topological defects such as entanglements and chain defects such as co-units that both prevent the chains to set up in crystallographic register despite good alignment with the microfibrils. This conclusion is consistent with the fact that thermal treatments do not significantly affect $D_{\mathrm{f}}$.

The question of the long period evolution is somewhat more complex. Figure 3 a shows that the fibrillar long period, $L_{\mathrm{pf}}$, is always lower than the initial long period, $L_{\mathrm{p}}$, in the limits of experimental accuracy. For annealed and isothermal samples, this finding may be rationalized in consideration that the draw temperature, $25^{\circ} \mathrm{C}<T_{\mathrm{d}}<100^{\circ} \mathrm{C}$, is always lower than the temperature of thermal treatment, $T_{\mathrm{c}}=$ $122^{\circ} \mathrm{C}$, so that one should expect $L_{\mathrm{pf}}<L_{\mathrm{p}}$ after melting-recrystallization (Figure $3 \mathrm{~b}$ ). However, for quenched samples (Figure 3a), it is hardly believable that the structural transformation could occur at a local temperature $T_{\mathrm{d}}{ }^{*}$ lower than the quenching temperature. This is especially true for $T_{\mathrm{d}}=100^{\circ} \mathrm{C}$. Moreover, self-heating is liable to increase the local temperature $T_{\mathrm{d}}{ }^{*}$ above the $T_{\mathrm{d}}$ value. Thereby, $T_{\mathrm{d}}{ }^{*}$ is likely to exceed the $T_{\mathrm{c}}$ of quenched samples so that melting-recrystallization cannot account for the finding $L_{\mathrm{pf}}<L_{\mathrm{p}}$.

One may thus propose a complementary mechanism to that of the model of Figure 7. One may consider that the melting point could not be reached during the transformation process, so that crystal destruction-recrystallization should be more appropriate than melting-recrystallization [11]. This implies that only a part of the crystalline phase should be only concerned by the phenomenon. This part should depend on the sample initial crystalline microstructure and chain topology, and experimental conditions as well.

Figure 8 shows a scenario of microfibril generation based on partial strain-induced crystal destruction- 


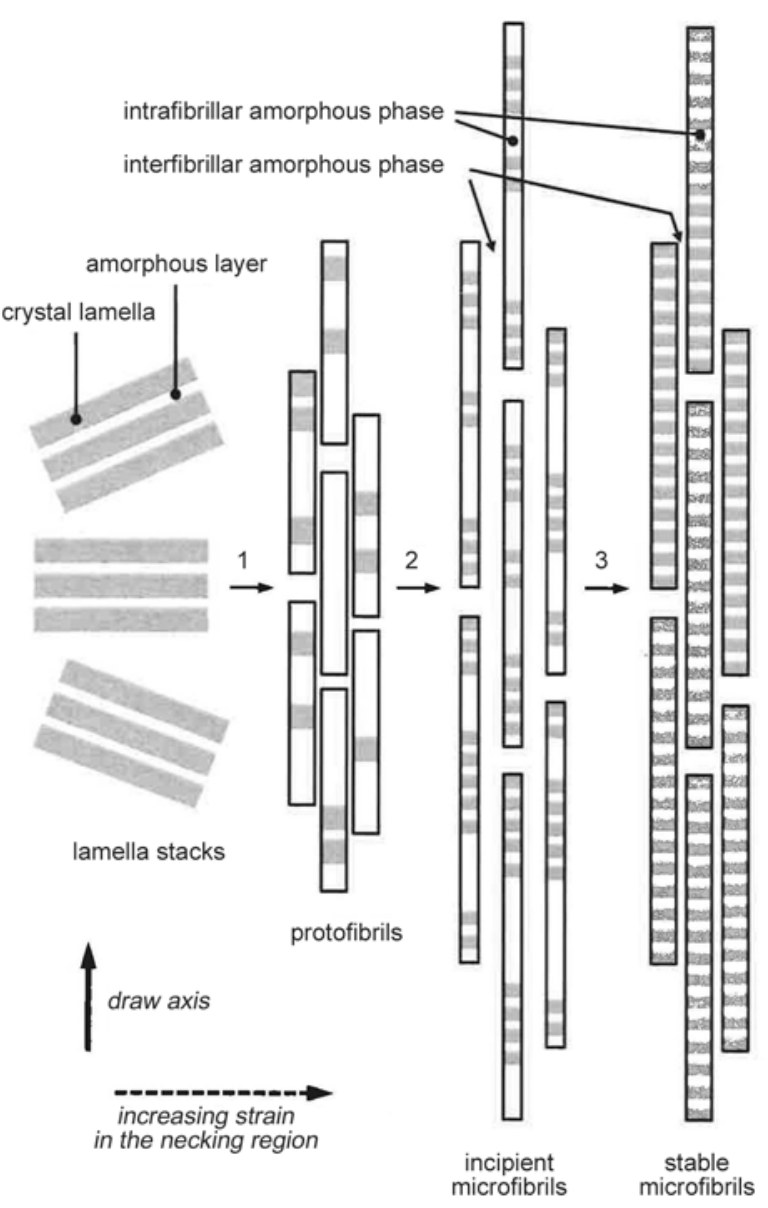

Figure 7. Schematic of the fibrillar transformation upon uniaxial drawing of polyethylene based on Peterlin's model including data from the present study (see text for details)

recrystallization. This model borrows from Thomas and coworkers observations $[45,53]$ regarding the fibrillar transformation of high density PE in relation to the chain topology, and the correlation with the natural draw ratio. Protofibrils (a) are first formed by fragmentation of the crystalline lamellae stacks. Then the stretched chains between the crystal fragments (b) in the protofibrils are prone to gradually crystallize benefiting from the strain-induced chain orientation. Then, stretching and self-heating promote chain unfolding and thinning down of the protofibrils (c) that gradually turn into microfibrils thanks to further crystallization and aggregation of unfolded chains (d). By this way, the final average $L_{\mathrm{pf}}$ value may be lower than the initial $L_{\mathrm{p}}$ value, although the local $T_{\mathrm{d}}{ }^{*}$ could be higher than $T_{\mathrm{c}}$. Besides, the amount of new crystal blocks grown under strain is likely to be lower for high $T_{\mathrm{d}}$ than for

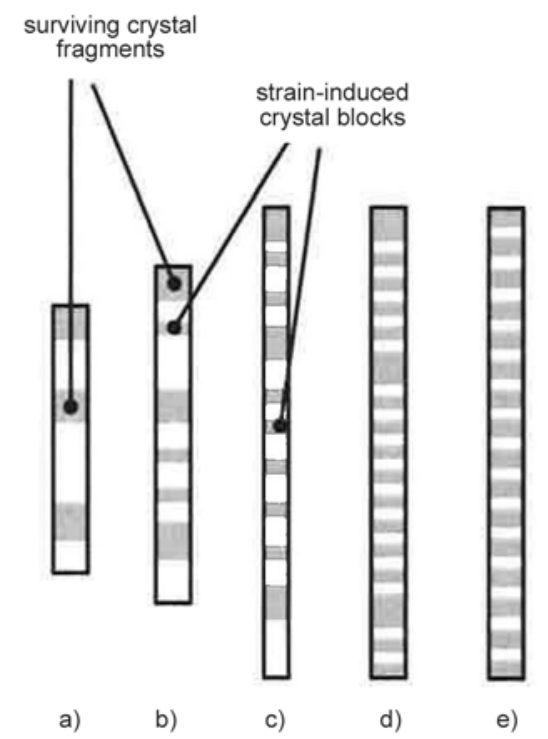

Figure 8. Alternative mechanism of microfibril generation in case of partial strain-induced melting (see text for detail)

low $T_{\mathrm{d}}$ owing to lesser density of critical nuclei. This could explain that $L_{\mathrm{pf}}$ is higher at high $T_{\mathrm{d}}$.

The presence of surviving crystal fragments with different crystal thickness than the strain-induced crystal blocks should disrupt the regular stacking in the microfibrils. As a matter of fact, none of the drawn samples displayed harmonics on the 2-streak SAXS patterns.

The amount of surviving crystal fragments may depend on both the sample initial crystalline microstructure and experimental conditions. High $T_{\mathrm{d}}$ and low initial $X_{\mathrm{c}}$ or $L_{\mathrm{p}}$ are favorable to crystal destruction owing to better chain relaxation in the crystalline phase. However, this quantity is hardly accessible to experiments.

The later refinement of the proposed fibrillar transformation model is perfectly consistent with the observation that $L_{\mathrm{pf}}$ turns roughly constant for $T_{\mathrm{d}} \leq$ $50^{\circ} \mathrm{C}$. Indeed, below the temperature of the crystal relaxation, molecular motions in the crystal are prohibited at usual tensile strain rates. Besides, considering that the $\beta$-relaxation in high density PE occurs at about $-40^{\circ} \mathrm{C}$ and the glass transition temperature at $-120^{\circ} \mathrm{C}$ [54], the molecular motions in the amorphous phase are little affected in the range $-40^{\circ} \mathrm{C}<$ $T_{\mathrm{d}}<50^{\circ} \mathrm{C}$. Therefore, the fibrillar transformation process should be practically insensitive to temperature in this $T_{\mathrm{d}}$ range. 


\section{Acknowledgements}

The authors are indebted to Total-Petrochemicals (Feluy, Belgium) for supplying the polymers and their molecular characteristics. The authors are also grateful to the European Synchrotron Radiation Facility (Grenoble, France) for time allocation on the BM02 beamline.

\section{References}

[1] Geil P. H.: Polymer single crystals. Wiley, New York (1973).

[2] Bigg D. M.: A review of techniques for processing ultra-high modulus polymers. Polymer Engineering and Science, 16, 725-734 (1976).

DOI: $10.1002 /$ pen. 760161103

[3] Zachariades A. E., Porter R. S.: The strength and stiffness of polymers. Marcel Dekker, New York (1983).

[4] Ward I. M.: The preparation, structure and properties of ultra-high modulus flexible polymers. Advances in Polymer Science, 10, 1-70 (1985).

DOI: $10.1007 / 3-540-15481-7 \quad 7$

[5] Lewis P. R.: High performance polymer fibres. Rapra, Shawburry (1997).

[6] Eichhorn S., Hearle J. W. S., Jaffe M., Kikutani M. E.: Handbook of textile fibre structure, Vol.1: Fundamentals and manufactured polymer fibres. Woodhead, Cambridge (2009).

[7] Statton W. O.: Polymer texture: The arrangement of crystallites. Journal of Polymer Science Part A: Polymer Chemistry, 41, 143-155 (1959).

DOI: $10.1002 /$ pol.1959.1204113811

[8] Peterlin A.: Folded chain model of highly drawn polyethylene. Polymer Engineering and Science, 9, 172-181 (1969). DOI: $10.1002 /$ pen.760090305

[9] Baltá-Calleja F-J., Peterlin A.: Plastic deformation of polypropylene. Part 2 The influence of temperature and draw-ratio on the axial long period. Journal of Materials Science, 4, 722-729 (1969).

DOI: $10.1007 / \mathrm{BF} 00742429$

[10] Sakaoku K., Morosoff N., Peterlin A.: Drawing of nylon 6 fibers (bristles). Journal of Polymer Science Part B: Polymer Physics, 11, 31-42 (1973).

DOI: $10.1002 /$ pol.1973.180110104

[11] Peterlin A.: Drawing and extrusion of semi-crystalline polymers. Colloid and Polymer Science, 265, 357-382 (1987).

DOI: $10.1007 / \mathrm{BF} 01412215$

[12] Séguéla R.: Plasticity of semi-crystalline polymers: Crystal slip versus melting-recrystallization. e-Polymers, no.32 (2007).

[13] Wu W., Wignall G. D., Mandelkern L.: A SANS study of the plastic deformation mechanism in polyethylene. Polymer, 33, 4137-4140 (1992).

DOI: $\underline{10.1016 / 0032-3861(92) 90617-6}$
[14] Annis B. K., Strizak J., Wignall G. D., Alamo R. G., Mandelkern L.: A small-angle neutron scattering study of the plastic deformation of linear polyethylene. Polymer, 37, 137-140 (1996).

DOI: 10.1016/0032-3861(96)81608-9

[15] Baer R. S., Bolduan O. E. A.: Diffraction by cylindrical bodies with periodic axial structure. Acta Crystallographica, 3, 236-241 (1950).

DOI: $10.1107 / \mathrm{S} 0365110 \times 50000586$

[16] Alexander L. E.: X-ray diffraction methods in polymer science. Wiley-Interscience, New York (1970).

[17] Murthy N. S., Bednarczyk C., Moore R. A. F., Grubb D. T.: Analysis of small-angle X-ray scattering from fibers: Structural changes in nylon 6 upon drawing and annealing. Journal of Polymer Science Part B: Polymer Physics, 34, 821-835 (1996).

DOI: 10.1002/(SICI)1099-0488(19960415)34:5<821:: AID-POLB1>3.0.CO;2-P

[18] Samon J. M., Schultz J. M., Hsiao B. S.: Study of the cold drawing of nylon 6 fiber by in-situ simultaneous small- and wide-angle X-ray scattering techniques. Polymer, 41, 2169-2182 (2000).

DOI: $10.1016 / \mathrm{S} 0032-3861(99) 00378-\mathrm{X}$

[19] Stribeck N.: Analysis of SAXS fiber patterns by means of projections. in 'Scattering from polymers' (eds.: Cebe P., Hsiao B. S., Lohs D. J.) ACS Symposium Series, Washington Vol 739, 41-56 (2000). DOI: $10.1021 / \mathrm{bk}-2000-0739 . c h 003$

[20] Ibanes C., David L., de Boissieu M., Séguéla R., Epicier T., Robert G.: Structure and mechanical behavior of nylon- 6 fibers filled with organic and mineral nanoparticles. I. Microstructure of spun and drawn fibers. Journal of Polymer Science Part B: Polymer Physics, 42, 3876-3892 (2004).

DOI: $10.1002 /$ polb.20217

[21] Peterlin A., Corneliussen A.: Small-angle X-ray diffraction studies of plastically deformed polyethylene. II. Influence of draw temperature, draw ratio, annealing temperature, and time. Journal of Polymer Science Part B: Polymer Physics, 6, 1273-1282 (1968). DOI: $10.1002 /$ pol.1968.160060706

[22] Peterlin A., Baltá-Calleja F-J.: Diffraction studies of plastically deformed polyethylene. Kolloid-Zeitschrift und Zeitschrift für Polymere, 242, 1093-1102 (1970). DOI: $10.1007 / \mathrm{BF} 02084715$

[23] Young R. J.: Dislocation model for yield in polyethylene. Philosophical Magazine, 30, 85-94 (1974). DOI: $10.1080 / 14786439808206535$

[24] Bowden P. B., Young R. J.: Deformation mechanisms in crystalline polymers. Journal of Materials Science, 9, 2034-2051 (1974). DOI: $10.1007 / \mathrm{BF} 00540553$

[25] Kajiyama T., Okada T., Takayanagi M.: The role of mosaic block structure on onset of deformation and fatigue of bulk crystallized polyethylene. Journal of Macromolecular Science Part B: Physics, 9, 35-69 (1974).

DOI: $\underline{10.1080 / 00222347408204539}$ 
[26] Lin L., Argon A. S.: Structure and plastic deformation of polyethylene. Journal of Materials Science, 29, 294 323 (1994).

DOI: $10.1007 / \mathrm{BF} 01162485$

[27] Butler M. F., Donald A. M., Ryan A. J.: Time resolved simultaneous small- and wide-angle X-ray scattering during polyethylene deformation: 1 . Cold drawing of ethylene- $\alpha$-olefin copolymers. Polymer, 38, 55215538 (1997).

DOI: 10.1016/S0032-3861(97)00111-0

[28] Butler M. F., Donald A. M., Ryan A. J.: Time resolved simultaneous small- and wide-angle X-ray scattering during polyethylene deformation - II. Cold drawing of linear polyethylene. Polymer, 39, 39-52 (1998). DOI: $10.1016 / \mathrm{S} 0032-3861(97) 00226-7$

[29] Hiss R., Hobeika S., Lynn C., Strobl G.: Network stretching, slip processes, and fragmentation of crystallites during uniaxial drawing of polyethylene and related copolymers. A comparative study. Macromolecules, 32, 4390-4403 (1999).

DOI: $10.1021 / \mathrm{ma9} 81776 \mathrm{~b}$

[30] Nitta K-H., Takayanagi M.: Role of tie molecules in the yielding deformation of isotactic polypropylene. Journal of Polymer Science Part B: Polymer Physics, 37, 357-368 (1999).

DOI: 10.1002/(SICI) 1099-0488(19990215)37:4<357:: AID-POLB9>3.3.CO;2-9

[31] Galeski A.: Strength and toughness of crystalline polymer systems. Progress in Polymer Science, 28, 16431699 (2003).

DOI: $10.1016 /$ j.progpolymsci.2003.09.003

[32] Plummer C. J. G.: Microdeformation and fracture in bulk polyolefins. Advances in Polymer Science, 169, 75-119 (2004).

DOI: $10.1007 / \mathrm{b} 13520$

[33] Pawlak A., Galeski A.: Plastic deformation of crystalline polymers: The role of cavitation and crystal plasticity. Macromolecules, 38, 9688-9697 (2005). DOI: $10.1021 / \mathrm{ma} 0508420$

[34] Séguéla R.: On the natural draw ratio of semi-crystalline polymers: Review of the mechanical, physical and molecular aspects. Macromolecular Materials and Engineering, 292, 235-244 (2007).

DOI: $10.1002 /$ mame.200600389

[35] Li Y., Iwakura Y., Shimizu H.: Polymer crystallites with few tie molecules from a miscible polymer blend. Macromolecules, 41, 3396-3400 (2008).

DOI: $10.1021 / \mathrm{ma} 800148 \mathrm{x}$

[36] Schneider K.: Investigation of structural changes in semi-crystalline polymers during deformation by synchrotron X-ray scattering. Journal of Polymer Science Part B: Polymer Physics, 48, 1574-1586 (2010). DOI: $10.1002 /$ polb.21971
[37] Humbert S., Lame O., Chenal J-M., Séguéla R., Vigier G.: Memory effect of the molecular topology of lamellar polyethylene on the strain-induced fibrillar structure. European Polymer Journal, 48, 1093-1100 (2012).

DOI: 10.1016/j.eurpolymj.2012.03.010

[38] Shroff R., Prasad A., Lee C.: Effect of molecular structure on rheological and crystallization properties of polyethylenes. Journal of Polymer Science Part B: Polymer Physics, 34, 2317-2333 (1996).

DOI: 10.1002/(SICI)1099-0488(199610)34:14<2317:: AID-POLB3>3.0.CO;2-T

[39] Hubert L., David L., Séguéla R., Vigier G., Degoulet C., Germain Y.: Physical and mechanical properties of polyethylene for pipes in relation to molecular architecture. I. Microstructure and crystallisation kinetics. Polymer, 42, 8425-8434 (2001).

DOI: 10.1016/S0032-3861(01)00351-2

[40] Xiong B., Lame O., Chenal J-M., Rochas C., Séguéla R., Vigier G.: Amorphous phase modulus and micromacro scale relationship in polyethylene via in situ SAXS and WAXS. Macromolecules, 48, 2149-2160 (2015).

DOI: 10.1021/acs.macromol.5b00181

[41] Wunderlich B.: Macromolecular physics, Vol.1: Crystal structure, morphology, defects. Academic Press, New York (1973).

[42] Humbert S., Lame O., Vigier G.: Polyethylene yielding behaviour: What is behind the correlation between yield stress and crystallinity? Polymer, 50, 3755-3761 (2009).

DOI: 10.1016/j.polymer.2009.05.017

[43] Guinier A., Fournet G.: Small-angle scattering of Xrays. Wiley, New York (1955).

[44] Ward I. M.: Mechanical properties of solid polymers. Wiley Interscience, New York (1980).

[45] Tarin P. M., Thomas E. L.: The role of inter- and intralinks in the transformation of folded chain lamellae into microfibrils. Polymer Engineering and Science, 19, 1017-1022 (1979). DOI: $10.1002 /$ pen.760191409

[46] Cazenave J., Séguéla R., Sixou B., Germain Y.: Shortterm mechanical and structural approaches for the evaluation of polyethylene stress crack resistance. Polymer, 47, 3904-3914 (2006).

DOI: 10.1016/j.polymer.2006.03.094

[47] Deblieck R. A. C., van Beek D. J. M., Ramerie K., Ward I. M.: Failure mechanisms in polyolefines: The role of crazing, shear yielding and the entanglement network. Polymer, 52, 2979-2990 (2011). DOI: $10.1016 /$ j.polymer.2011.03.055

[48] Xiong B., Lame O., Chenal J. M., Rochas C., Séguéla R., Vigier G.: In-situ SAXS study and modeling of the cavitation/crystal-shear competition in semi-crystalline polymers: Influence of temperature and microstructure in polyethylene. Polymer, 54, 5408-5418 (2013). DOI: $10.1016 /$ j. polymer.2013.07.055 
[49] Men Y., Rieger J., Strobl G.: Role of the entangled amorphous network in tensile deformation of semicrystalline polymers. Physical Review Letters, 91, 095502/1095502/4 (2003).

DOI: 10.1103/PhysRevLett.91.095502

[50] Wang Y., Jiang Z., Wu Z., Men Y.: Tensile deformation of polybutene-1 with stable form I at elevated temperature. Macromolecules, 46, 518-522 (2013). DOI: $10.1021 / \mathrm{ma302389j}$

[51] Lu Y., Wang Y., Fu L., Jiang Z., Men Y.: Crystallization, recrystallization, and melting lines in syndiotactic polypropylene crystallized from quiescent melt and semicrystalline state due to stress-induced localized melting and recrystallization. Journal of Physical Chemistry, 118, 13019-13023 (2014).

DOI: $10.1021 / j p 5093702$
[52] Nitta K-H., Takayanagi M.: Tensile yield of isotactic polypropylene in terms of a lamellar-cluster model. Journal of Polymer Science Part B: Polymer Physics, 38, 1037-1044 (2000).

DOI: $10.1002 /($ SICI) 1099-0488(20000415)38:8<1037 $\because$ AID-POLB4>3.0.CO;2-R

[53] Adams W. W., Yang D., Thomas E. L.: Direct visualization of microstructural deformation processes in polyethylene. Journal of Materials Science, 21, 2239-2253 (1986).

DOI: $10.1007 / \mathrm{BF} 01114262$

[54] Stehling F. C., Mandelkern L.: The glass temperature of linear polyethylene. Macromolecules, 3, 242-252 (1970). DOI: $10.1021 / \mathrm{ma} 60014 \mathrm{a} 023$ 